\title{
Seminarios Virtuales. Cómo Aplicarlos en Educación a Distancia
}

\author{
(Virtual Seminars. How to Use them en Distance Education)
}

\author{
Wolfram Laaser \\ FernUniversität Hagen
}

(Alemania)

\begin{abstract}
RESUMEN: El artículo trata de un elemento de la educación a distancia que hasta hace poco era ofracido sólo por la enseñanza presencial en cursos de corta duración. Este elemento es tradicionalmente etiquetado como "seminario", lo que difiere de una clase o de una "unidad didáctica" en que aquel está basado en contribuciones propias de los alumnos y en la discusión académica. Hoy en día se han levado a cabo varios intentos de organizzar también seminarios 'online'. Las especiales características y dificultades son discutida en detalle en este artículo. Igualmente se analizan las posibles soluciones técnicas. Finalmente se argumenta que los seminarios virtuales tiene características especiales que han de ser investigadas más a fondo y que pueden complementar más que substituir a los encuentros cara a cara.
\end{abstract}

\section{Seminarios Virtuales - FernUniversität - Características Especiales}

ABSTRACT: The article deals with an element in distance education which has been offered so far only through face to face teaching in residential short term courses. This element is traditionally labeled as "seminar" which differes from the lecture or "unidad didactica" in that it is based on students own contributions and on academic discussion. Today various attempts are made to deliver seminars as well online. The special characteristics and difficulties are discussed here in detail. Also alternative technical solutions are analyzed. At the end it is argued that virtual seminars have special characteristics that still have to be further investigated and that may rather complement than substitute face to face meetings.

Virtual Seminars - FernUniversität - Special Characteristics 


\section{LOS SEMINARIOS VIRTUALES YA SON UNA REALIDAD EN EL MARCO DE LA FERNUNIVERSITÄT DE HAGEN}

Tanto en las conferencias nacionales como en las internacionales se presenta frecuentemente la posibilidad de llevar a cabo seminarios virtuales como una innovación; pues este tipo de seminario ya es una realidad cotidiana dentro del media mix de nuestro sistema de educación a distancia universitaria.

Brindemos algunos ejemplos concretos. El primer catedrático de nuestra universidad que ofreció un seminario virtual es titular de una cátedra en filosofía. Este hecho a primera vista parece sorprendente porque los filósofos, por regla general, no son especialistas en el uso de Internet. Por otro lado, la citada materia está basada en la palabra escrita, pero también en la discusión académica. Por ello, a la postre, la selección de este campo fue menos casual de lo que parecía al principio. Otros tópicos de seminarios virtuales eran por ejemplo el teletrabajo, "mobbing" (Cátedra de Psicología Organizacional), aprendizaje colaborativo en redes (Cátedra de Ciencias de la Computación) y uso de medios en educación a distancia (Centro de Desarrollo de Educación a Distancia). Algunos de estos seminarios se dirigían a estudiantes de las materias respectivas mientras que otros tenían como objetivo el entrenamiento de educadores a distancia. Ahora bien, antes de discutir los modelos y opciones que se presentaron en estos seminarios queremos determinar con mayor precisión lo que podemos entender bajo el término Seminario Virtual.

\section{2. ¿CUÁLES SON LAS DIFERENCIAS ENTRE SEMINARIOS VIRTUALES Y SEMINARIOS CONVENCIONALES?}

En nuestra tradición académica los seminarios para la educación universitaria son estructurados de manera que los participantes deben presentar breves trabajos escritos basados en sus lecturas e investigaciones individuales. Después el estudiante tiene que discutir la exposición con los docentes y también con los demás estudiantes. Al final su contribución será evaluada y calificada. Los seminarios normalmente se llevan a cabo en una fecha determinada, con horarios fijos en un determinado local físico. Este tipo de seminario está considerado habitualmente como elemento indispensable de la enseñanza universitaria. Por estas razones también forma parte de la enseñanza en cursos a distancia en el ámbito universitario.

Por otro lado, esta manera de enseñar cara a cara no se corresponde con otras características de la educación a distancia como son el estudio independiente y flexible en términos de tiempo y espacio.

¿Cuáles son en comparación las características de los seminarios virtuales? La presentación de artículos y de otros resultados elaborados por parte de los estudiantes serán comunicados a través de redes electrónicas. También la discusión sobre estos será efectuada vía redes. En gran medida esta comunicación será asincrónica, aunque también puede ser sincrónica, es decir, en tiempo real. El lugar del seminario virtual es Internet. Los participantes se comunican a través de sus computadoras personales 
conectadas a Internet desde la casa o el trabajo, o sea, del centro regional de estudios de la universidad. A causa de la asincronicidad de la comunicación, los seminarios virtuales, frecuentemente, exigen una duración más larga del seminario entero comparado con los seminarios convencionales presenciales.

\section{UN SEMINARIO VIRTUAL EN DETALLE}

Para explicar cómo llevamos a cabo los seminarios virtuales queremos referir brevemente algunas experiencias con un seminario sobre el tema "Mobbing" ofrecido por la Cátedra de Psicología Organizacional que fue realizado en el año 1997. El seminario empezó en abril y continuó hasta julio del mismo año. El cuerpo docente proponía las fases siguientes como esquema del seminario:

Fase 1: Cuestiones de Organización.

Fase 2:Introducción al tema "Mobbing" (tópicos y conceptos). Este tema debía ser preparado por los estudiantes en forma de trabajo de grupo.

Fase 3:Modelos, teoría y resultados de lecturas e investigaciones (ponencias presentadas por los estudiantes).

Fase 4: Métodos de intervención (lectura de artículos y reuniones de CHAT para su discusión).

Fase 5:Prevención del Mobbing y Perspectivas ( lectura de artículos y reunión de CHAT final "on line").

En este seminario se establecieron "grupos de noticias" como medio principal de comunicación. Los grupos de noticias servían para distribuir ponencias y otras lecturas así como para comunicar resultados del trabajo de grupo. Además formaron la plataforma para las discusiones entre estudiantes y entre alumnos y el cuerpo docente. Para organizar y canalizar los diferentes tipos de comunicación se formaron tres grupos de noticias diferentes. El primero se llamó "moderación". El acceso a éste fue restringido a los docentes que participaban y se dedicaba a mensajes dirigidos a los estudiantes. El segundo grupo se denominó “discusión". Este quedó abierto a todos los participantes para discutir cuestiones relacionadas directamente con los contenidos y tópicos del seminario. Un tercero se llamó "café" y éste no fue supervisado directamente por los profesores. Aquí el objetivo era facilitar la comunicación más informal entre alumnos. 


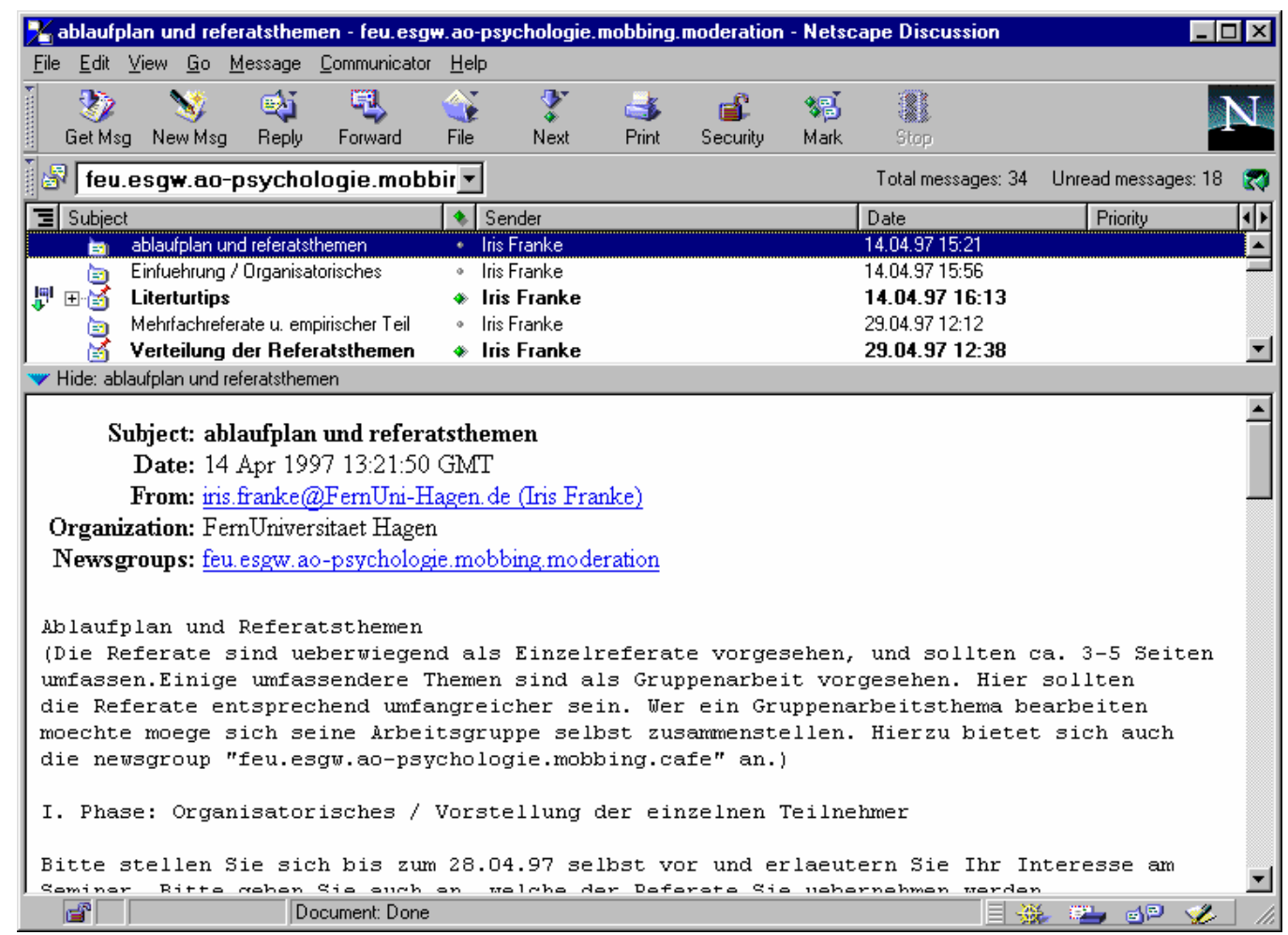

A continuación se referirán algunos comentarios individuales como se los podía encontrar típicamente en los diferentes grupos de noticias mencionados arriba. Habitualmente al inicio de un seminario presencial los participantes tienen que presentarse brevemente. Esto también es parte de un seminario virtual. Una alumna escribió lo siguiente:

"Me llamo Silke S., tengo 28 años, soy casada y vivo en Hamburgo. Estudio a tiempo parcial a distancia y estoy cursando el cuarto año de filosofía. Además hago cursos de psicología y de nueva literatura alemana. Mi ocupación principal es el cuidado de mis dos hijas (4 y 2 años). Durante los fines de semana trabajo en un restaurante Mc Donald. Probablemente es una de las razones por las que me interesa el tema del 'Mobbing'. Por otro lado creo que también me animó la posibilidad de obtener un certificado de un seminario que me permitiera estudiar desde mi casa. En cuanto a mi contribución en grupos de trabajo me interesaba el tópico 8. Además me gustaría trabajar sobre el último tema "Estado del Arte de las investigaciones sobre 'Mobbing'”.

Comentarios similares muestran claramente que los seminarios virtuales son un elemento muy apreciado por nuestra clientela. Otra observación interesante fue que en el ámbito del seminario virtual la formación de grupos estudiantiles de trabajo colaborativo parecía aún más fácil que en los seminarios presenciales correspondientes. Puede ser que el impacto emocional no tan inmediato sea responsable de tal fenómeno. Hemos descubierto efectos similares en que los estudiantes mismos favorecen la comunicación mediatizada cuando llevamos a cabo exámenes orales a través de videoconferencias. 
Al respecto queremos citar otro comentario que se refiere a la ponencia de un coparticipante:

"Hola, Kristina. He encontrado una referencia bibliográfica sobre "mobbing en niños" en B. Zuschlag, Mobbing. En esta fuente también hay otras referencias. El hecho de que dediquemos un $30 \%$ de nuestra vida al trabajo asalariado me asustó totalmente, pero como esto está mencionado en varios libros tengo que creerlo. Si en el futuro trabajáramos más años, los puestos con salarios elevados serían cada vez más escasos. Tal vez por este motivo, la lucha y competencia entre los jóvenes se está incrementando y con este fenómeno también aparece posiblemente el 'mobbing'“.

El comentario anterior muestra un aspecto típico de la comunicación electrónica. Es el estilo de comunicación menos formal. Además un solo mensaje muchas veces contiene una variedad de temas y asuntos diferentes (mensajes multipropósito), por ejemplo, avisos técnicos o indicaciones acerca de dónde consiguir materiales referentes al tema, reacciones emocionales sobre una respuesta o comentarios a un aspecto académico. Los comentarios reflejados hasta el momento fueron sacados del grupo de noticias "discusión". El próximo comentario refleja el papel que el docente cumple a través del grupo "moderación".

"Estimados participantes: para mejorar nuestra comunicación en el seminario reflexionen ustedes sobre los puntos siguientes: ¿Qué no he entendido bien? ¿En qué no estoy de acuerdo con una presentación? ¿En qué me gustaría ampliar o contribuir de alguna manera? Otro punto: Por favor, indicar en la parte superior a qué documento se refiere así como el tipo de comentario“.

Mensajes que incluyen varios asuntos y la enorme cantidad de textos para ser leídos son un factor que complica bastante la organización y el manejo del seminario y por ello merece atención especial. Una medida para canalizar y hacer llegar la mayor información a los grupos, es aprovechando esta información y formando subgrupos, por ejemplo con informadores elegidos o nombrados de entre los estudiantes que coordinarán los subgrupos y, más adelante, comunicarán la síntesis al plenario.

El tercer tipo de noticias denominado "café" esta dedicado y reservado para contactos y observaciones informales. Estos comentarios pueden tener relevancia también para el transcurso y éxito de un seminario virtual. Aquí se discuten asuntos técnicos, se intercambian direcciones de correo electrónico, se establecen referencia a páginas Web o se ponen de acuerdo sobre el transporte para asistir a una reunión. Para los docentes puede ser aconsejable echar una ojeada de vez en cuando a este grupo de comentarios para ver cómo está el clima entre los participantes y cuáles son sus preocupaciones.

\section{ALGUNOS ASPECTOS DIDÁCTICOS}

Conceptos y esquemas didácticos desarrollados para seminarios presenciales en gran medida son aplicables también a seminarios virtuales. Como la cohesión (sentido de pertenecer a un grupo) entre los participantes en un seminario virtual normalmente será menos fuerte comparado con seminarios presenciales, parece importante aplicar 
aquellas estructuras que fortalecen e incentivan el trabajo grupal. En varios seminarios, específicamente los llevados a cabo por la Facultad de Educación, se exigía a los estudiantes un trabajo grupal, por ejemplo, desarrollar un cuestionario y hacer una prueba con los otros participantes para evaluar más adelante las respuestas. Este tipo de actividad favorece la posibilidad de practicar y compartir lo aprendido o elaborado por sus colegas sin fases de intervención continua por parte del docente, lo que parece aprovechar realmente las características del seminario virtual. También, didácticamente, este esquema puede resultar más rico que la pura presentación de ponencias con discusión posterior como se acostumbra en el seminario convencional. Queda la pregunta de cómo evaluar la actividad del estudiante individualmente. En este aspecto se recurre también a un trabajo adicional, escrito y elaborado individualmente, pero en la nota final se toman en cuenta también las otras actividades del estudiante en el transcurso del seminario.

Otro punto sería cómo reducir posibles barreras emocionales al inicio del seminario. También en este caso alguna actividad común puede ayudar. Otro medio bastante adecuado sería determinar una fecha temprana para una conferencia textual on line (Chat), porque aquí el diálogo es más directo y vivo, lo que facilita "romper el hielo". En la comunicación demorada (delayed communication) muchas veces los estudiantes tienen que esperar mucho tiempo hasta que reciben una respuesta directa. Esto contribuye al anonimato, a la sobrecarga de información inespecífica y prolonga la duración total de los seminarios virtuales. Además, tenemos que tomar en cuenta que la comunicación por escrito es más lenta que la comunicación oral. Finalmente falta la imagen, los gestos y la voz para descifrar e interpretar los comentarios. Todos estos aspectos pueden reducir participaciones espontáneas y resultar en una conducta más bien pasiva (lurking). Si es posible, organizar videoconferencias en ciertas etapas del seminario también puede elevar la motivación y el entusiasmo de los participantes. Sin embargo, debido a las restricciones que implican las conferencias simultáneas técnica y económicamente, así como en términos de flexibilidad y ritmo individual, estos elementos no son la forma predominante de comunicación. También debe considerarse que el costo de la comunicación on line es más elevado.

Un elemento interesante para detectar cómo se sienten los estudiantes en el seminario actual fue lo que se denomina "virtual flashligt" (instantánea virtual). Esta consiste simplemente en solicitar, de vez en cuando, a los estudiantes, interrumpir momentáneamente su trabajo con los contenidos y comunicar su estado de ánimo en ese momento. A continuación se recoge una respuesta a dicha petición:

“Hola. Me gustaría mostraros una 'instantánea'. Como este es mi primer seminario virtual no tenía muchas expectativas concretas, pero este seminario ha resultado requerir mucho más trabajo de lo que yo me imaginaba. Las ponencias necesitan mucho tiempo de lectura, igual que los diferentes comentarios. A esto se suma la elaboración de la propia ponencia. También problemas técnicos consumen mucho tiempo disponible. Aunque muchos textos pueden leerse 'off-line' por medio del 'downloading', así se reduce el costo de la comunicación telefónica. Creo, en fin, que puede organizarse el seminario en una forma más estructurada“. 
Debido a la ausencia del contacto cara a cara y a la falta de gestos y respuestas inmediatas, deben buscarse formas alternativas para controlar y dirigir el transcurso del seminario. Por otro lado, la comunicación escrita ofrece posibilidades para hacer más estricto el diálogo académico entre los participantes. Pueden formularse las contribuciones con más cuidado y precisión. Además los todos comentarios están documentados y son, así, repetibles en cualquier momento.

\section{ASPECTOS TÉCNICOS}

La primera cuestión por resolver es qué formato dar a las principales contribuciones del seminario, artículos y otros resultados.

Una forma relativamente sencilla de hacer llegar los textos a los participantes sería la de enviar estos textos directamente mediante los grupos de noticias en formato ASCII. Un problema que puede ocurrir con esta modalidad es que se pierde el formato del texto, la forma de los diagramas, la representación de fórmulas matemáticas u otros elementos más especiales. Si nosotros exigimos que los artículos sean legibles e imprimibles sin pérdidas es preferible partir de archivos de WORD. Más aún, para evitar problemas de impresión, puede ser aconsejable transformar los archivos de WORD a través de un lector pdf tipo ACROBAT a un archivo pdf. Como resultado se mantiene el fotmato como fue creado por el autor y la impresión no se altera independientemente del tipo de impresora local.

Otra posibilidad serÍa la transferencia de los archivos vía FTP. Los archivos se ubican como archivos UNIX en un servidor UNIX y los estudiantes pueden acceder el servidor FTP desde su casa. Otra posibilidad es colocar los textos en páginas HTML del World Wide Web. En este caso los archivos WORD pueden ser convertidos a HTML, lo que es técnicamente bastante cómodo y factible directamente dentro del programa WORD. Las páginas del Web serán entonces leídas con un lector (browser) HTML. Sin embargo, puede perderse una parte del formato al imprimir los archivos.

Finalmente las contribuciones pueden ser enviadas como ficheros adjuntos de correo, lo que es una manera de distribución relativamente simple y poco complicada. En caso de que se distribuyan los archivos con extensión pdf debe subministrarse también un lector para este tipo de archivos, por ejemplo el ACROBAT READER que está disponible gratuitamente.

¿Cuál seria el modelo más adecuado? Depende de las exigencias por parte de los organizadores del seminario así como de los hábitos y del equipamiento del que disponen los estudiantes.

También será necesario tomar una decisión sobre cuál sería la plataforma para llevar a cabo las discusiones electrónicas. En la actualidad los grupos de noticias son relativamente populares. Sin embargo no tienen protección. La intimidad de la discusión (privacy) no está garantizada y participantes o comentarios no deseados no pueden ser excluidos por los organizadores. Técnicamente los participantes tienen que tener acceso a los grupos de noticias a través de un proveedor de Internet. Esto debe verificarse antes 
de iniciar el seminario, porque no todos los centros de cómputo universitario ofrecen un servidor para NEWS.

Una plataforma alternativa es la distribución de comentarios por medio de listas de distribución. Estas pueden ser protegidas y los organizadores tienen así mayor control sobre la distribución de los mensajes. Si no se disponede un sistema de conferencia por computadora, la estructuración y organización de los comentarios puede resultar más complicada, mientras en el sistema de Grupos de Noticias la organización de éstos es relativamente fácil.

Una tercera opción es la conversión de los comentarios enviados por correo electrónico al formato HTML, para luego colocarlos en páginas Web. También para este propósito existen paquetes de software que facilitan esta tarea. También en este caso la organización de los comentarios (referencing) puede resultar menos amigable.

Para reducir el costo de la comunicación 'on-line' puede recurrirse a lectoras 'off-line' como lo es por ejemplo el programa FreeAgent. Así, todos los comentarios se cargan primeramente en el disco duro para leerlos después 'off-line'. Ahora este tipo de servicio también está ofrecido con los nuevos Navegadores de Microsoft Explorer o de Netscape.

Para mostrar cómo pueden integrarse todos los servicios diferentes en una sola plataforma queremos presentar como modelo las paginas Web que desarrollamos para el Centro para el Desarrollo de la Educación a Distancia cuando ofrecimos en 1996 un curso de perfeccionamiento para docentes provenientes del campo de la educación a distancia. El home-page del seminario que nosotros denominamos "coloquio virtual" mostraba los siguientes elementos de navegación:

Informaciones sobre el seminario (inscripción, tópicos, etc.).

unformaciones actuales (sesiones de chat, modificaciones del programa).

u Contribuciones (artículos presentados como archivos pdf).

w Participantes (vínculos de correo electrónico y direcciones de instituciones de empleo).

u Tutores (breve presentación de cada tutor en una página del web con vínculo para correo electrónico).

$u$ Discusión (acceso directo a los grupos de noticias).

u Aplicaciones para cargar (ejemplo: aplicaciones para chat).

u Glosario (téminos técnicos usados en los seminarios; formato pdf). 


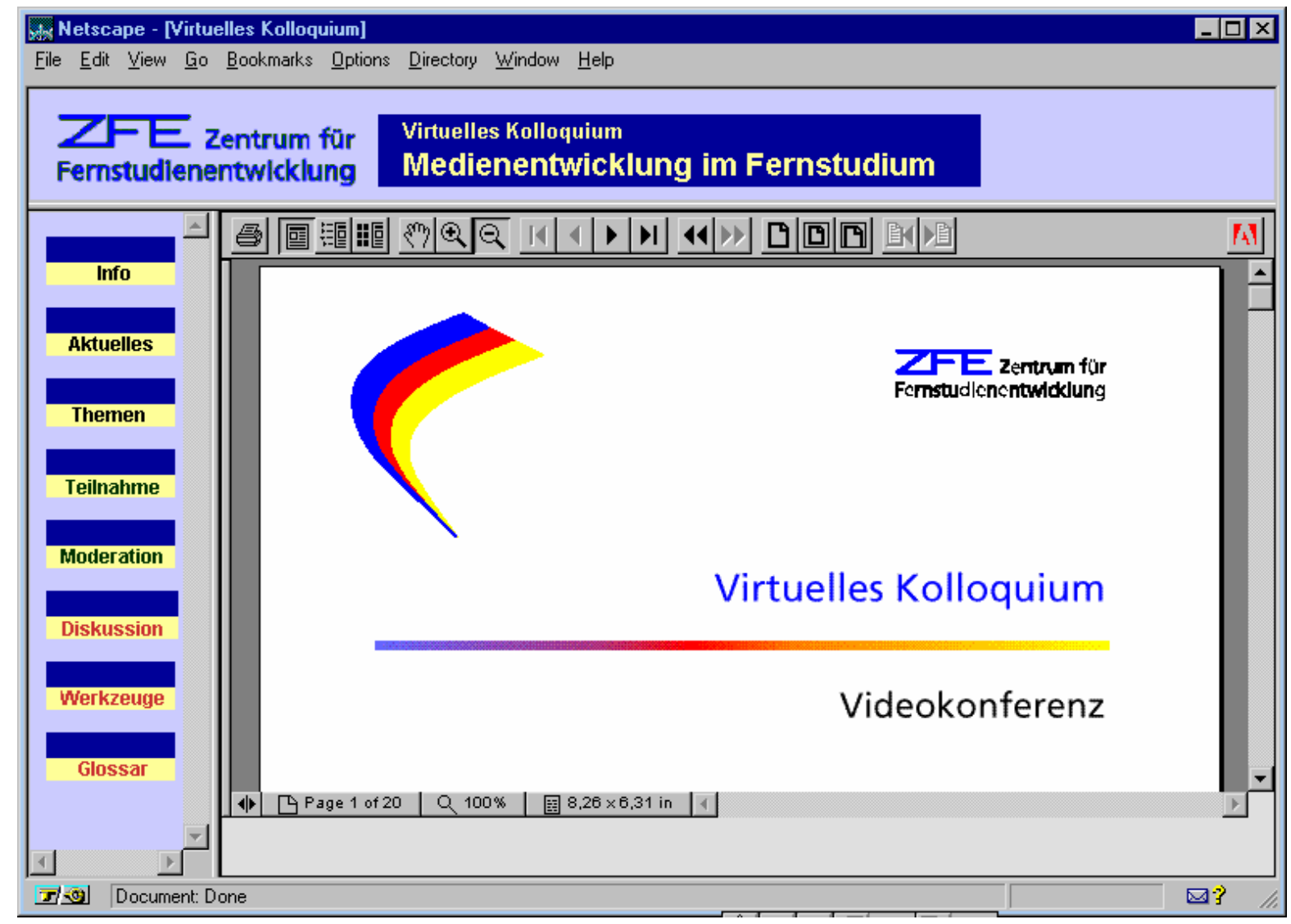

El segundo ejemplo para una plataforma de un seminario virtual fue tomado de un seminario sobre "trabajo cooperativo en redes" de la Cátedra de Informática. Se puede observar como opciones de navegación:

Marco a la izquierda: Opciones para navegar dentro de la universidad virtual u Marco abajo:
o Introducción
o Tópicos
o Organización
- Trabajos de los participantes
o Comunicación entre los participantes
o Participantes

Dentro de los marcos vemos la página que se refiere a los trabajos estudiantiles con las categorías:

o Denominación del tema

o Grupos de Noticias para discutir sobre este tema

o Participantes del grupo estudiantil

o Estructuración del tema

o Vínculos para cargar el resultado del trabajo cooperativo o lectura online 
o Fechas de reuniones de CHAT

o Comentarios adicionales

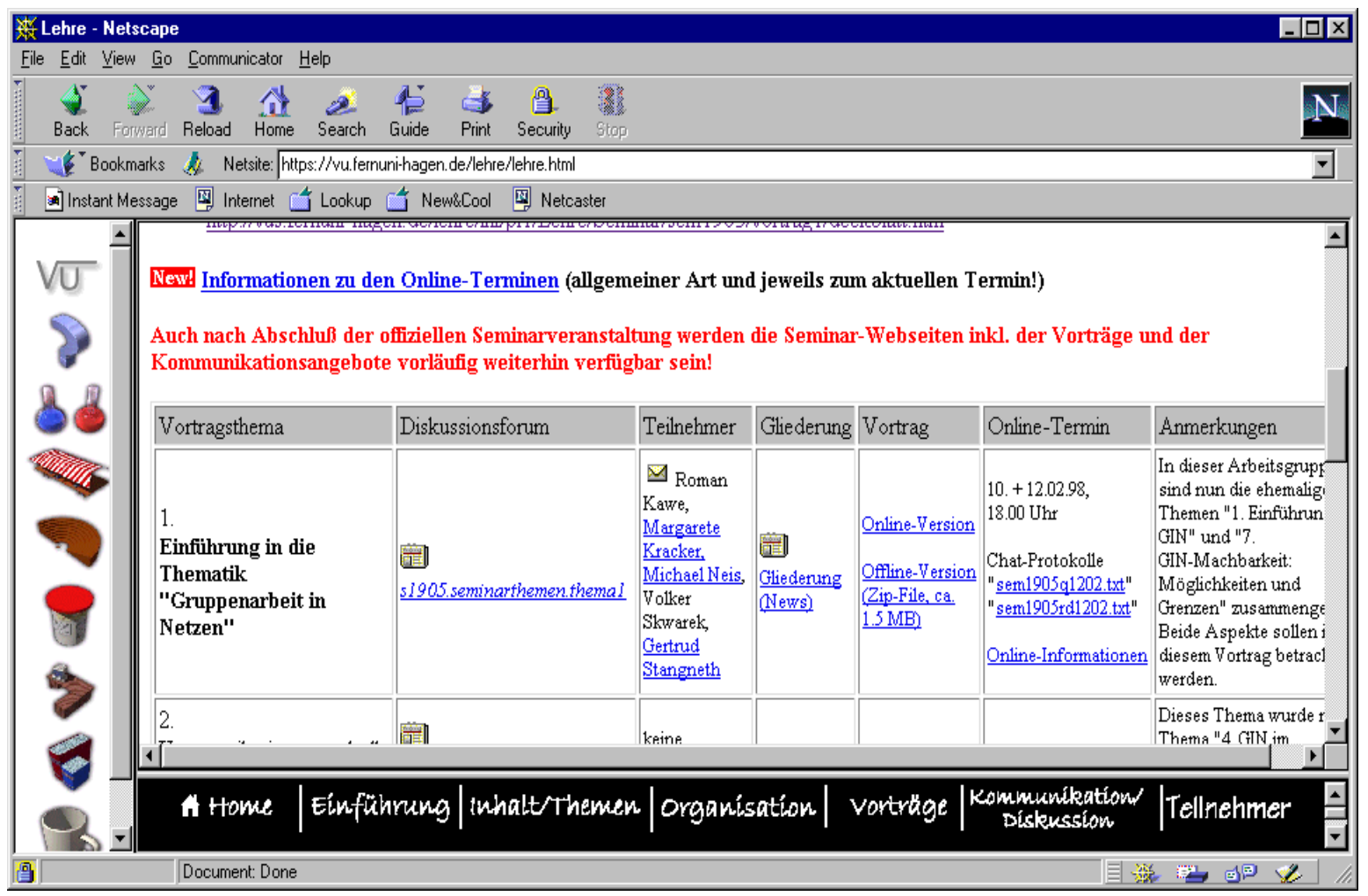

Es obvio que este tipo de integración en una plataforma amigable facilita considerablemente la navegación dentro del ámbito del seminario virtual.

\section{EVALUACIÓN DE SEMINARIOS VIRTUALES}

Es obvio que se puede evaluar seminarios virtuales con toda la batería de instrumentos clásicos de las ciencias sociales. Pero la pregunta más atractiva es lo que está detrás de esto. Un producto prácticamente automático de la comunicación electrónica es el registro de todas las acciones de los participantes y con esto también el registro de los comentarios escritos. Este material, muy amplio, puede ser analizado para generar nuevos conocimientos sobre esta nueva modalidad dentro del campo de la educación a distancia. Ya se encuentran numerosas investigaciones comparando la cantidad y la calidad de mensajes en este modelo con el modelo del seminario presencial, aún es discutible, sin embargo, si la comparación de ambas formas de seminarios realmente es lo que se debe analizar. Más importante parece descubrir y definir las nuevas características del medio en sí.

En un seminario sobre metodología de las ciencias sociales (de marzo a septiembre de 1996) se intercambió, por ejemplo, el siguiente número de mensajes: 


$\begin{array}{lll}\text { Mensajes recibidos } & \text { Correo Eletrónico } & \text { Grupos de Noticias } \\ \text { Participantes } & 166 & 281 \\ \text { Organizadores } & 121 & 191 \\ \text { Total } & 287 & 472\end{array}$

Fuente: H. Heidbrink, Ein virtuelles Methodenseminar an der FernUniversität, en: Internet für Psychologen (B. Batinic Ed.), Hogrefe, Göttingen 1997, P. 403

El ejemplo muestra la enorme cantidad de comentarios. Como cada comentario es registrado palabra por palabra incluyendo incluso los diálogos de conferencias simultáneas (chat), esto posibilita un análisis cualitativo en una medida anteriormente desconocida. Contenido, interpretación, tamaño, frecuencia, estilo, destinatarios sólo son algunos criterios para ser investigados. A estas áreas se suman cuestiones más técnicas como tiempo y duración de las conexiones y costo de éstas.

\section{NUEVAMENTE “SEMINARIOS VIRTUALES VERSUS PRESENCIALES”}

Obviamente los seminarios virtuales, en su preparación inicial, implican mucho más trabajo tanto para los organizadores como para los participantes. Por otro lado, es obvio también que la oferta de seminarios tradicionales en el campo de la educación a distancia será siempre limitado en áreas lejanas o donde no hay tutores cualificados. El costo del traslado de docentes o de estudiantes tiene que ser comparado con el costo del equipamiento técnico (aunque no se suelen comprar computadoras sólo para participar en seminarios virtuales) y, sobre todo, con el costo de conexión.

Aparte de las consideraciones de flexibilidad y de los aspectos económicos debemos tomar en cuenta que siempre la recepción oral es más rápida que la lectura de textos. Las discusiones mediante textos requieren referencias claras para seguir más fácilmente las complicadas o enredadas estructuras de la comunicación electrónica. Por otro lado la comunicación textual puede ser aún más precisa que la comunicación oral. Un déficit de los seminarios virtuales es la participación pasiva de gran parte de los participantes. Sin embargo, esta frecuente participación pasiva, ("lurking"), no necesariamente tiene que ser vista como completamente negativa. Algo similar ocurre en el seminario convencional, en el cual los estudiantes pueden aprovechar los comentarios y las preguntas de sus colegas ("witness learning").

Los seminarios virtuales, por lo tanto, son una nueva alternativa que permite varias opciones interesantes, como por ejemplo, incluir charlas de personas invitadas, la participación de expertos o el uso amplio de referencias a los enormes recursos del WEB por medio de hipervínculos. Este último es una forma de hacer referencia a materiales que están igualmente disponibles para todos los participantes

Finalmente los seminarios virtuales proporcionan flexibilidad de tiempo al equipo docente y a los estudiantes que participan. Sin embargo, para mantener una retroalimentación, no demasiado demorada, es aconsejable dar a la comunicación un ritmo adecuado y estructurar el seminario mediante algunas actividades fijas (fechas para terminar ciertas tareas, reuniones de chat, etc.). 
Los seminarios virtuales son una modalidad específica y diferente de los seminarios presenciales. Las características específicas impiden considerarlos como sustitutos equivalentes de los seminarios presenciales. Si sus características son aprovechadas y sus limitaciones bien entendidas, esta modalidad puede enriquecer marcadamente el espectro de los medios disponibles en programas de educación a distancia.

\section{REFERENCIAS BIBLIOGRÁFICAS}

C. Bremer, Gruppenbasiertes vernetztes Lernen im Internet, GMW Forum 1-2, 1998, P. 11-16

H. Fritsch, Host contacted, waiting for reply, FernUniversität Hagen, Mayo 1997

W. J. Gibbs, J. E. McKenrick, Virtual Courses: Delivering Courses On-Line, paper presented to the ICDE Worldconference, PennState University 1997

H. Heidbrink, Ein virtuelles Methodenseminar an der FernUniversität, en: Internet Psychologen (B. Batinic Ed.), Hogrefe, Göttingen 1997

M. Herberger et al, Collaborative Learning via WWW in Legal Education, in: The Journal of Information, Law and Technology (JILT) 1988 (2)

W. Laaser, Virtual Colloquy on the Internet, en: Journal of Research in Educational Media, Indian Council for Research in Educational Media (Ed.), 4 (1) 1997, P. 43-49

W. Laaser, Videoconferencias como recurso suplementario en sistemas de educación a distancia, en: Revista Iberoamericana de Educación a Distancia, Vol. IV, Nr. 3, 1994, P. 39-49

S. Schwan, Media characteristics and knowledge acquisition in computer conferencing, Deutsches Institut für Fernstudienforschung Ed., Tübingen 1997

\section{PERFIL ACADÉMICO-PROFESIONAL DEL AUTOR}

El Dr. Wolfram Laaser es el Director Académico del Centro para el Desarrollo de la Educación a Distancia de la FernUniversität de Hagen, Alemania.

Dr. Wolfram Laaser

FernUniversität Hagen

Zentrum für Fernstudienentwicklung (ZFE)

In der Krone, 17

Hagen D-58084

Deustchland/Alemania

Tfno.: 004923319874210

Fax: 00492331987194210

Dirección Electrónico: wolfram.laaser@fernuni-hagen.de 